Research article

\title{
Genomic diversity of citrate fermentation in Klebsiella
} pneumoniae

Ying-Tsong Chen ${ }^{\dagger 1}$, Tsai-Lien Liao ${ }^{\dagger 1}$, Keh-Ming Wu ${ }^{\dagger 1,2}$, TsaiLing Lauderdale ${ }^{3}$, Jing-Jou Yan ${ }^{4}$, I-Wen Huang 3 , Min-Chi Lu' ${ }^{5}$, Yi-Chyi Lai ${ }^{5}$, Yen-Ming Liu ${ }^{1}$, Hung-Yu Shu ${ }^{6}$, Jin-Town Wang ${ }^{7}$, Ih-Jen Su ${ }^{3}$ and ShihFeng Tsai $* 1,2,8$

Address: ${ }^{1}$ Division of Molecular and Genomic Medicine, National Health Research Institutes, Miaoli, Taiwan, Republic of China, ${ }^{2}$ Genome Research Center and Institute of Biomedical Informatics, National Yang-Ming University, Taipei, Taiwan, Republic of China, ${ }^{3}$ Division of Infectious Diseases, National Health Research Institutes, Zhunan, Miaoli, Taiwan, Republic of China, ${ }^{4}$ Department of Pathology, National Cheng Kung University Hospital, Tainan, Taiwan, Republic of China, ${ }^{5}$ College of Medicine, Chung Shan Medical University, Taichung, Taiwan, Republic of China, ${ }^{6}$ Department of Bioscience Technology, Chang Jung Christian University, Tainan County, Taiwan, Republic of China, ${ }^{7}$ Department and Graduate Institute of Microbiology, National Taiwan University, Taipei, Taiwan, Republic of China and ${ }^{8}$ Department of Life Sciences and Institute of Genome Sciences, National Yang-Ming University, Taipei, Taiwan, Republic of China

Email: Ying-Tsong Chen - onion@nhri.org.tw; Tsai-Lien Liao - tsailien@nhri.org.tw; Keh-Ming Wu - kmwu@nhri.org.tw; TsaiLing Lauderdale - Lauderdale@nhri.org.tw; Jing-Jou Yan - jingjou@mail.ncku.edu.tw; I-Wen Huang - yvone@nhri.org.tw; MinChi Lu - luminchi@seed.net.tw; Yi-Chyi Lai - yclai@csmu.edu.tw; Yen-Ming Liu -ymliu@nhri.org.tw; Hung-Yu Shu - hyshu@mail.cjcu.edu.tw; Jin-Town Wang - wangit@ntu.edu.tw; Ih-Jen Su - suihjen@nhri.org.tw; Shih-Feng Tsai* - petsai@nhri.org.tw

* Corresponding author †Equal contributors

Published: 15 August 2009

BMC Microbiology 2009, 9:168 doi:10.1186/147|-2180-9-168
Received: 30 April 2009

Accepted: 15 August 2009

This article is available from: http://www.biomedcentral.com//47/-2/80/9//68

(c) 2009 Chen et al; licensee BioMed Central Ltd.

This is an Open Access article distributed under the terms of the Creative Commons Attribution License (http://creativecommons.org/licenses/by/2.0), which permits unrestricted use, distribution, and reproduction in any medium, provided the original work is properly cited.

\begin{abstract}
Background: It has long been recognized that Klebsiella pneumoniae can grow anaerobically on citrate. Genes responsible for citrate fermentation of $K$. pneumoniae were known to be located in a I3-kb gene cluster on the chromosome. By whole genome comparison of the available $K$. pneumoniae sequences (MGH 78578, 342, and NTUH-K2044), however, we discovered that the fermentation gene cluster was present in MGH 78578 and 342, but absent in NTUH-K2044. In the present study, the previously unknown genome diversity of citrate fermentation among $K$. pneumoniae clinical isolates was investigated.

Results: Using a genomic microarray containing probe sequences from multiple $K$. pneumoniae strains, we investigated genetic diversity among $K$. pneumoniae clinical isolates and found that a genomic region containing the citrate fermentation genes was not universally present in all strains. We confirmed by PCR analysis that the gene cluster was detectable in about half of the strains tested. To demonstrate the metabolic function of the genomic region, anaerobic growth of $K$. pneumoniae in artificial urine medium (AUM) was examined for ten strains with different clinical histories and genomic backgrounds, and the citrate fermentation potential was found correlated with the genomic region. PCR detection of the genomic region yielded high positive rates among a variety of clinical isolates collected from urine, blood, wound infection, and pneumonia. Conserved genetic organizations in the vicinity of the citrate fermentation gene clusters among K. pneumoniae, Salmonella enterica, and Escherichia coli suggest that the I3$\mathrm{kb}$ genomic region were not independently acquired.
\end{abstract}


Conclusion: Not all, but nearly half of the K. pneumoniae clinical isolates carry the genes responsible for anaerobic growth on citrate. Genomic variation of citrate fermentation genes in $K$. pneumoniae may contribute to metabolic diversity and adaptation to variable nutrient conditions in different environments.

\section{Background}

Citrate, a ubiquitous natural compound that exists in all living cells, can be used by several enterobacterial species as a carbon and energy source. Klebsiella pneumoniae has been known to be able to grow anaerobically with citrate as the sole carbon source. During the past decade, the physiology, biochemistry, and regulation of this pathway have been extensively studied in K. pneumoniae [1-4]. The fermentation process involves uptake of citrate by a $\mathrm{Na}+$ dependent citrate carrier, cleavage into oxaloacetate and acetate by citrate lyase, and decarboxylation of oxaloacetate to pyruvate by oxaloacetate decarboxylase. Finally, pyruvate can be converted to acetate, formate and carbon dioxide by means of anaerobic pyruvate catabolism.

Genes responsible for citrate fermentation of K. pneumoniae can be identified in a 13 -kb gene cluster on the chromosome [[2,5], and this study]. These genes are contained within two divergently transcribed operons, citC2D2E2F2G2 and citS-oadGAB-citAB [6]. The citC2D2E2F2G2 operon encodes the citrate lyase ligase, the $\gamma_{-}, \beta-$, and $\alpha$-subunits of citrate lyase, and triphosphoribosyl-dephospho-coenzyme A synthase. The citsoadGAB (dcoCAB)-citAB operon encodes the citrate carrier Cits, the $\gamma_{-}, \alpha_{-}$, and $\beta$-subunits of oxaloacetate decarboxylase, and the citrate-sensing CitA-CitB two component system [5]. Transcription at the promoters in front of the two operons is activated by phospho-CitB and Crp-cAMP [2]. Additionally, citX, which is required for synthesis of the citrate lyase prosthetic group, has been identified in a second genomic location along with citW, a putative citrate transporter gene, and citYZ that encodes a two component system homologous to CitA-CitB [7]. The citWX genes and the divergent citYZ are adjacent but placed in opposite directions.

Coliform organisms, especially E. coli and K. pneumoniae, are the most common causes of urinary tract infection. Uropathogenic pathogens have been studied extensively for virulence factors such as the fimbriae and adhesins $[8,9]$. These virulence factors facilitate the anchorage of the pathogens to the extracellular matrix of the bladder and urinary tract, and thus prevent them from being washed out by the urine. Type I pili, which is produced by all members of the Enterobacteriaceae family, has long been implicated as an important virulence factor in mediating $K$. pneumoniae urinary infection $[10,11]$. Alternatively, the ability to grow in urine may be important for the persistence of pathogens in the urinary tract. Except for trace of amino acids, citrate is the only carbon source available in normal human urine.

In K. pneumoniae, little has been reported about the genomic basis for nutrient growth. We recently completed the whole-genome sequence of NTUH-K2044 (GenBank accession no. AP006725) [12], a K. pneumoniae strain isolated from the blood of a previously healthy individual who was diagnosed with a community-acquired primary liver abscess and metastatic meningitis [13]. By comparison with the available genome sequences of the other $K$. pneumoniae strains, MGH 78578 (GenBank: CP000647), and 342 (GenBank: CP000964) [14], we discovered that the entire $13-\mathrm{kb}$ chromosomal region carrying the aforementioned citrate fermentation genes in MGH 78578 and 342 was missing in NTUH-K2044. We postulated that the 13 -kb genomic region containing genes for citrate fermentation might facilitate the use of urine citrate in oxygenlimited or anaerobic conditions, and thus, permit the growth of $K$. pneumoniae in the urinary tract. To test this hypothesis, an artificial urine medium (AUM) designed to provide controlled composition of the human urine [15] was used in this study to ensure reproducibility. The correlation between presence/absence of the citrate fermentation genes and anaerobic growth in this system was investigated. The distribution of the citrate fermentation genes among different $K$. pneumoniae clinical isolates was also analyzed.

\section{Results and Discussion}

The citrate fermentation genes in a I3-kb genomic region Located at $2791640906 \mathrm{bp}$ in the genomic sequence of $K$. pneumoniae strain MGH 78578, the 13-kb citrate fermentation gene locus contains 11 orfs, which constitute two divergently transcribed operons citC2D2E2F2G2 and citSoadGAB (dcoCAB)-citAB (Figure 1). The organization of these genes is the same as in the recently published $K$. pneumoniae 342 genome [14]. The dihydrodipicolinate reductase gene $d a p B$ and the hypothetical orfs located at the two ends of the 13-kb region in the MGH 78578 and 342 genomes are next to each other in the NTUH-K2044 genome. Missing in the corresponding location, the citrate genes are nowhere found in the NTUH-K2044 genome, and the region is replaced by a 155-bp non-coding sequence. Since many genomic or pathogenicity islands found in bacteria genomes were associated with tRNA genes, we also tried to look for tRNA genes at the edge of 
$\mathrm{a}$
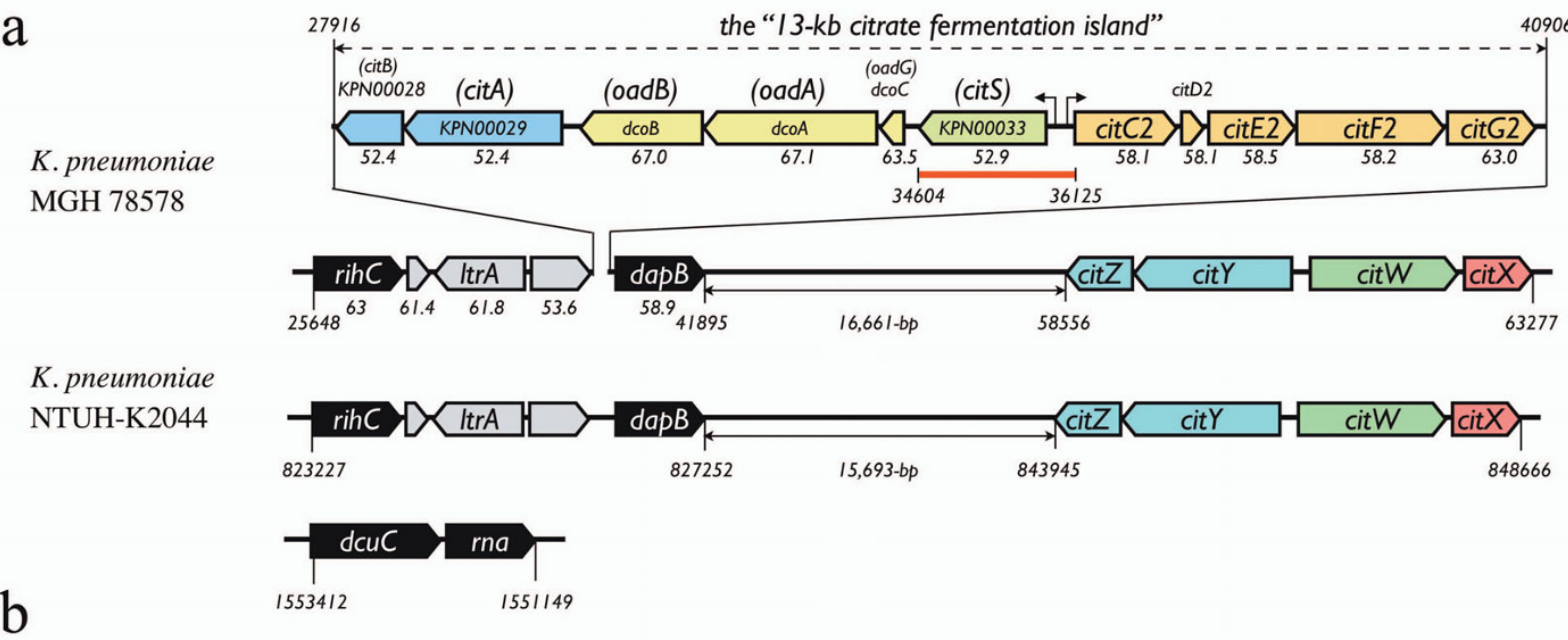

S. enterica LT2

(citB) (citA)

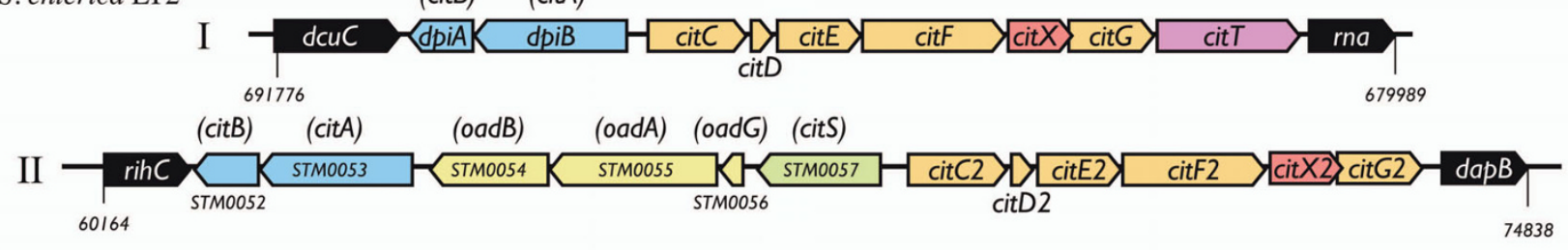

C

E. coli $\mathrm{K} 12$

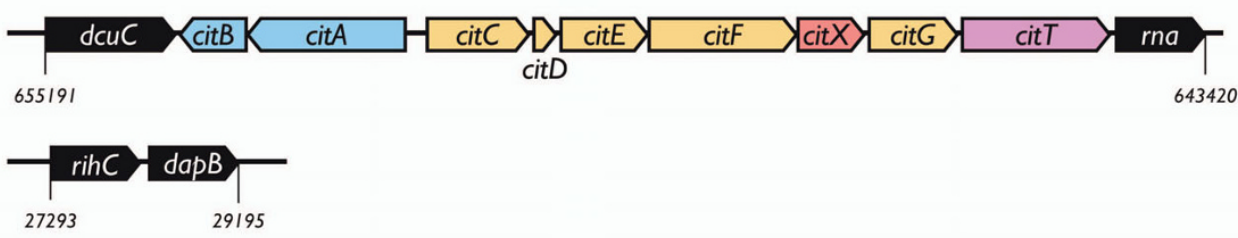

Figure I

Comparative analysis of citrate fermentation gene locus. The I3-kb genomic region is present in $\mathrm{K}$. pneumoniae $\mathrm{MGH}$ 78578 but absent in NTUH-K2044 (a). The location of the I3-kb genomic region for citrate fermentation, which includes two divergently transcribed operons, citS-oadGAB-citAB and citC2D2E2F2G2, are marked. The adjacent hypothetical orfs are shown in gray, among which the ItrA encodes a putative transcriptional regulator. The citYZ-citWX gene clusters downstream of the dapB in both MGH 78578 and NTUH-K2044 are also depicted. The G+C value for each orf in MGH 78578 is shown below each orf. The red bar indicates the corresponding location replaced by an apramycin resistant gene in the promoter knockedout strain, NK8- $\Delta$ cit, derived from the NK8 clinical strain. Corresponding citrate fermentation loci from S. enterica serovar Typhimurium LT2 and E. coli KI2 are shown (b and c) with colours indicating homologous genes. Alternative gene names in parentheses on top of some orfs for better comparison were based on homology search. The locations of these regions in the genomes are marked below. In the LT2 genome, two clusters of citrate fermentation genes were found. The corresponding flanking genes for locus I, dcuC and rna, and locus II, rihC and dapB, are shown in black.

this region. However, it appeared that the $13-\mathrm{kb}$ genomic region carrying the citrate fermentation genes is not located within or near any tRNA gene, nor does it contain any direct repeat or known mobility sequence. This is in agreement with a recent study of bacterial genome flux, which indicated that, among twenty Escherichia coli genomes, many of the integration hotspots are not necessarily recombinogenic [16].
Another gene cluster containing the citWX and the divergent citYZ genes are conserved among $K$. pneumoniae genomes (Figure 1a). In NTUH-K2044, the citWX-citYZ gene cluster is located at 15,693-bp downstream of the dapB. The existence of this additional gene cluster, especially the cit $X$, is important for the function of citrate lyase in K. pneumoniae. Unlike the counterpart identified in Salmonella enterica (Figure $1 \mathrm{~b}$ ), the $13-\mathrm{kb}$ region in K. pneu- 
moniae does not contain citX for the biosynthesis of the prosthetic group of citrate lyase [7]. In MGH 78578, the deduced amino acid sequences of cit $Y$ and citZ are 43\% and $41 \%$ identical to CitA and CitB, respectively.

\section{Nearly half of the K. pneumoniae clinical isolates carry the I3-kb genomic island}

The presence/absence of the 13-kb region was investigated in additional $K$. pneumoniae clinical isolates (NK3, NK5, NK6, NK8, NK9, NK25, NK29, NK245, CG43, CMKa01 through CMKa08, CMKa10). These isolates were collected from patients with pneumonia (3), bacteremia (4), liver abscess (7), UTI (2), meningitis (1), and endophthalmitis (1). We conducted comparative genomic hybridization (CGH) analysis on the test strains with custom-made DNA microarray (NimbleGen), in which a total of 389,266 probes were designed based on the CDSs of five sequenced K. pneumoniae genomes [12]. For the current report, we have analyzed the results of the predicted coding sequences spanning the 13-kb region of MGH 78578. As shown in Figure 2, each of the 19 strains (including MGH 78578 as a control) was compared against the NTUH-K2044 reference genome. The dots represent the DNA copy number log ratios between the reference and tested genomes for the 687 probes corresponding to the sequences spanning the 13-kb region. Since the NTUHK2044 genome does not carry the cit genes, these results indicate that the 9 strains with dots plotted at the baseline in this region (NK5, NK6, NK9, CG43, CMKa01, CMKa02, CMKa04, CMKa08, and CMKa10) do not carry these genes in their genomes. The other ten strains shown in below, including MGH 78578, gave higher signals for the cit genes than that from the reference (Figure 2). By contrast, all strains have the same copy number of the flanking genes (rihC, hypothetical orfs, and $d a p B$ ) as the NTUH-K2044.

The oad genes within the 13-kb region are missing in NTUH-K2044, but the strain possesses an additional copy of oad genes at the tartrate-fermentation gene cluster outside this region. In contrast, according to the genomic sequence, MGH 78578 (GenBank: CP000647) carries three copies of the oad genes, including one in the 13-kb region. This is also confirmed by the CGH result, which indicated that four strains, MGH 78578, NK8, CMKa05, and $\mathrm{CMKa07}$, carry more than one copy of the oad genes and showed higher signal in the oad-probed region. On the other hand, CMKa10, NK5 and CG43, do not have oad genes and were represented by CGH plots below the baseline. We conclude that the $13-\mathrm{kb}$ citrate fermentation gene sequence is not a uniform feature of $K$. pneumoniae and that the oadGAB gene copy number is variable among the analyzed strains.

In a recent report, it is shown that all $K$. pneumoniae strains could grow on citrate as sole carbon source when tested aerobically [17]. A stark contrast is the ability of $K$. pneumoniae to grown on citrate anaerobically. While all $K$. pneumoniae isolates can grow on citrate aerobically, our results suggested that only about half of them carry the 13$\mathrm{kb}$ gene cluster for anaerobic citrate utilization.

\section{The I3-kb genomic island permits anaerobic growth in artificial urine}

As citrate is a major carbon source in human urine, we then asked whether the 13-kb genomic island could contribute to $K$. pneumoniae growth in the urinary tract. Although human urine is a suitable culture medium, the urine constituents can vary considerably between individuals under different conditions. It has been reported that the dissolved oxygen (DO) in urine is about $4.2 \mathrm{ppm}$, which is also variable and mainly reflects the renal metabolic state [18]. In patients with urinary infections, the urine DO is significantly reduced as a result of oxygen consumption by the microbes [18]. Therefore, in this study an artificial urine medium (AUM) developed to provide an experimental condition similar to that of the human urine [15] was used. To simulate growth conditions in the urinary tract, $K$. pneumoniae isolates were cultured in AUM at $37^{\circ}$ under oxygen-deprived condition.

Notable difference in the growth curves was observed when K. pneumoniae clinical strains were cultured anaerobically in AUM. After 27 hours incubation, five strains with the 13-kb genomic island (NK3, NK8, NK25, NK29, NK245), showed significant growth in AUM $\left(\mathrm{OD}_{600}\right.$ : $0.170 .43)$. In contrast, little growth $\left(\mathrm{OD}_{600}: 0.040 .06\right)$ was detected for strains that do not have the 13-kb genomic island (NTUH-K2044, NK5, NK6, NK9, CG43). The turbidities $\left(\mathrm{OD}_{600}\right)$ of NK8 and NTUH-K2044 at different time points during the 27-hour incubation in AUM were also measured. Note that little growth was detected in NTUH-K2044, a strain that lacks the citrate fermentation gene cluster (Figure 3 ), while exponential logarithmic phase growth was observed from 15 to $19 \mathrm{~h}$ in the NK8 strain that carries the 13-kb genomic island (Figure 4).

To demonstrate that the citrate fermentation genes present in the 13-kb region have allowed alternative use of carbon and energy source, a fosmid, F06C06, which contains the entire 13-kb region from NK8, was transformed into NTUH-K2044. As shown in Figure 3, this fosmid enabled the bacteria (NTUH-K2044-F06C06) to grow anaerobically in AUM. The logarithmic growth (from 11 to 15 h) of the fosmid-transformed clone was shifted to the left and the cells reached the stationary phase earlier than that of the NK8. This may be a result of gene copy number discrepancies between the fosmid transformants and NK8, or a result of other genetic factors specific to the NTUHK2044 genome. Similarly, the F06C06 fosmid sequence enabled the anaerobic growth of E. coli epi300 (Epicenter Technologies, Madison, WI) transformants in AUM (data 


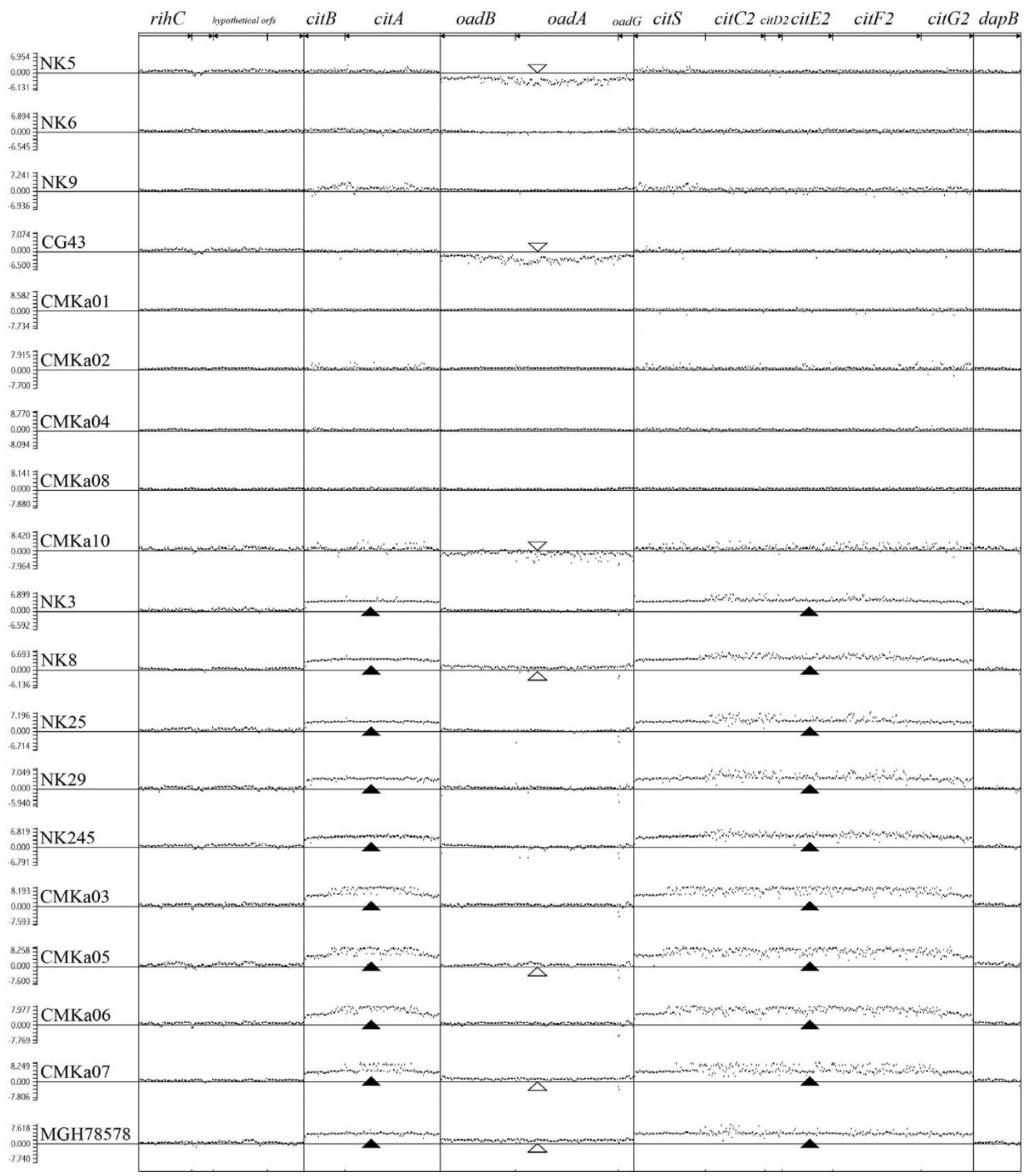

\section{Figure 2}

Genomic variation at the citrate fermentation gene locus. Divergence of the I3-kb genomic region in $19 \mathrm{~K}$. pneumoniae strains was detected by CGH analysis using the NimbleGen chips. Hybridization signals of each probes placed in the order of the MGH 78578 genome were compared with those of the reference strain, NTUH-K2044. The probes covering the cit genes and the oad genes of the 13-kb region were shown together with that of the adjacent orfs. The normalized CGH signals for each probe are plotted as black dots. The dot position above or under the baseline represents higher or lower copy of specific genomic sequence in comparison to the reference. The scores in vertical axis are log2 values of test/reference signal intensity obtained from image scanning of hybridization results. The detection of elevated scores in the cit genes (citA-B, citS citG2) in the last 10 strains (from NK3 to MGH 78278) is marked by solid triangles. Variations in the oad region are marked by open triangles. 


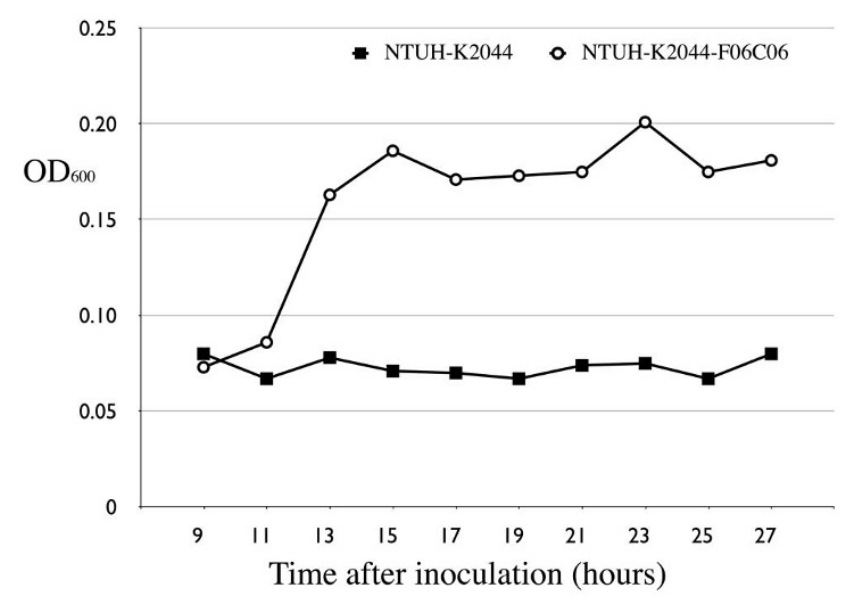

Figure 3

Citrate gene cluster permits fermentation growth in AUM for the NTUH-K2044 strain. NTUH-K2044, a strain that lacks the I3-kb genomic region; NTUH-K2044F06C06, NTUH-K2044 transformed by a fosmid (F06C06) carrying the I3-kb genomic region responsible for citrate fermentation from NK8.

not shown). As a control, the $K$. pneumoniae strains NTUH-K2044, NK8, NTUH-K2044-F06C06, and NK8$\Delta$ cit were cultured anaerobically in AUM medium prepared without citrate, all four strains showed no sign of growth in 27 hours.

To demonstrate that an intact citrate gene cluster is necessary for anaerobic growth, we created by homologous

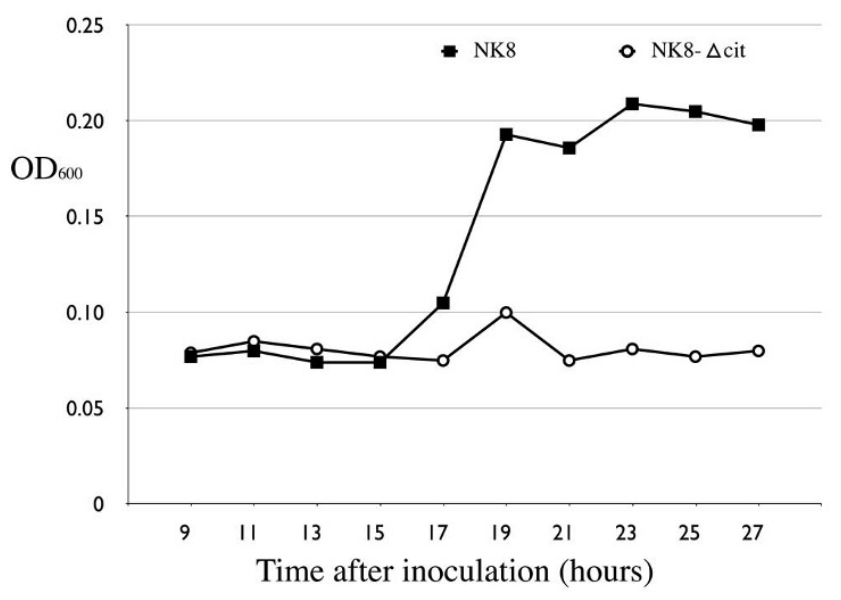

\section{Figure 4}

Citrate gene cluster is necessary for fermentation growth in AUM for the NK8 strain. NK8 is a clinical strain carrying the same citrate fermentation genes as the sequenced reference strain, MGH 78578; NK8- $\Delta$ cit, NK8 with the $13-\mathrm{kb}$ genomic region disrupted at the promoter region. The initial $O D_{600}$ of the inoculated $A U M$ culture is 0.0005 . recombination a genetic mutant in which the entire citS and the nearby citC2 promoter was replaced with an apramycin resistance gene. The citS-citC2 intergenic region contains binding sites for the response regulator CitB and cyclic AMP receptor protein (CRP), which mediates catabolic repression of citrate fermentation genes under anaerobic conditions [4]. The gene disruption was confirmed by PCR and sequencing of the region. The corresponding location of the altered sequence in the citrate fermentation island is indicated in Figure 1a. As consistent with the fact that the citC2 and citS promoters control the expression of the citC2D2E2F2G2 and citS-oadGABcit $A B$ operons, disruption of this regulatory region in the resultant strain, NK8- $\Delta$ cit, crippled its ability to grow anaerobically in AUM $\left(\mathrm{OD}_{600}=0.042\right.$ after 27 -h incubation) (Figure 4). Taken together, our data support that the citrate fermentation island permits and is necessary for anaerobic growth of $K$. pneumoniae in AUM using citrate as the sole carbon source.

\section{Citrate fermentation gene cluster in K. pneumoniae clinical isolates}

From the genetic studies on the citrate fermentation in AUM, it seems plausible that the ability of K. pneumoniae to grow in urine may provide the organism an added advantage in urinary tract infections (UTI), thus a higher percentage of citrate fermentation genomic island-positive K. pneumoniae strains would be expected in urine isolates than in non-urine isolates. To test this hypothesis, a total of $187 \mathrm{~K}$. pneumoniae clinical isolates collected from urine and non-urine specimens including blood, respiratory tract, wound, bile, ear, eye, and IV catheters, were analyzed for the presence of the $13-\mathrm{kb}$ island by using 5 PCR primer pairs designed across the region (Table 1). As shown in Table 2, 55 out of the 93 (59\%) urine isolates carried the genomic island, while 53/94 (56.3\%) of nonurine were test positive for the gene cluster. Thus, we did not find apparent correlation between the possession of the $13-\mathrm{kb}$ genomic region and urinary tract infection in this case collection.

In uropathogenetic E. coli strains, adhesins enable the anchorage to urinary tract to overcome the hydrodynamics of micturition, even though E. coli cannot live solely on citrate in anaerobic condition [2]. Other factors in the $K$. pneumoniae genome likely also contribute to urinary infection. To investigate the host-microbial interaction in UTI and to overcome the complex clinical situations, animal models will be necessary for determining the role of this 13 -kb genomic island in K. pneumoniae in colonizing the urinary tract.

\section{Genomic diversity on citrate fermentation}

The genes associated with citrate fermentation are different in composition and order in the sequenced Enterobacteriaceae genomes (Figure 1). In Salmonella enterica serovar 
Table I: Primer pairs used for detecting citrate fermentation genes.

\begin{tabular}{|c|c|c|c|}
\hline \multicolumn{2}{|c|}{ Primer sequences } & \multirow{2}{*}{$\begin{array}{c}\text { Genes covered } \\
\text { citA, citB }\end{array}$} & \multirow{2}{*}{$\begin{array}{c}\text { Product size (bp) } \\
952\end{array}$} \\
\hline I. & 5'-CCGGGCCTGAATATTAAACA-3' & & \\
\hline & 5'-CAACAGCAGTCGGAAAGTCA-3' & & \\
\hline \multirow[t]{2}{*}{2.} & 5'-GGATCTTCCGCTCCTTATCC-3' & oadA, oadB & 890 \\
\hline & 5'-GGAAGCCATGAAGATGGAGA-3' & & \\
\hline \multirow[t]{2}{*}{3.} & 5'-GCCCATCAGGATAGTTGGAA-3' & $\operatorname{citS}, \operatorname{cit} C 2$ & 970 \\
\hline & 5'-CAGCTCATAGGCCAGTGTCA-3' & & \\
\hline \multirow[t]{2}{*}{4.} & 5'-CGATGTGATGGTCAGGATTG-3' & citD2, citE2 & 770 \\
\hline & 5'-CGGGCGTAGAACAGTTCAGT-3' & & \\
\hline \multirow[t]{2}{*}{5.} & 5'-CATCGATGTGATTCGTCAGG-3' & citF2, citG2 & 873 \\
\hline & 5'-GCAATCAGCTCATCGTCAAA-3' & & \\
\hline
\end{tabular}

Typhimurium LT2 (GenBank: AE006468), which is capable of citrate fermentation using the same pathway, two gene clusters similar to the $13-\mathrm{kb}$ region are present in the genome (Figure $1 \mathrm{~b}$ ). One of them (locus I) showing similar gene arrangement ( $c i t A B$, and divergent citCDEFXGT) was identified between the rna RNase I gene (Locus_tag: STM0617, location: 679989680795) and the $d c u C$ C4dicarboxylate transporter gene (Locus_tag: STM0627, location: 690391691776) in the LT2 genome. The other (locus II) (citS-oadGAB-citAB, and divergent citC2D2E2F2X2G2) was found between rihC putative nucleotide hydrolase gene (Locus_tag: STM0051, location: 6016461084) and dapB (Locus_tag: STM0064, location: 7401774838 ). Both loci in LT2 carry the cit $X$ gene in respect to that of the $13-\mathrm{kb}$ island of K. pneumoniae. Based on the composition of the gene clusters and the genes at the vicinity, it appears that the second copy (locus II) from LT2 is more related (closer) to the 13-kb island of K. pneumoniae, albeit three hypothetical orfs (Figure 1a) next to the citB in K. pneumoniae are missing in LT2. The first copy of the gene cluster from LT2, as shown in Figure 1b, is similar in gene organization to the citrate fermentation gene cluster in E. coli K12 (GenBank: $\underline{\mathrm{U} 00096}$ ), which contains a citAB and a divergent citCDEFXGT positioned next to the rna RNase I gene (Locus_tag: b0611, location: 643420644226) (Figure 1c). The citT encodes a citratesuccinate antiporter for citrate uptake in E. coli [19]. While the citrate fermentation genes corresponding to locus I is missing in K. pneumoniae, homologs of the rna and $d c u C$ identified at the two ends of this gene cluster were juxtaposed to each other in the K. pneumoniae NTUH-K2044 (KP1607 and KP1608, location: 15511491553412), MGH 78578 (location: 742196744459 ) and 342 (location: 29622033964466). On the other hand, homologs of the rih $C$ and $d a p B$, the genes flanking the two ends of the $13-\mathrm{kb}$ genomic island from $K$. pneumoniae, were found adjacent to each other in the E. coli K12 genome (Locus_tag: b0030 and b0031, location: 2729329295).

In the MGH 78578, three oad gene clusters were found, one located in the 13-kb citrate fermentation gene cluster, another located at the downstream of the galETKM genes for galactose metabolism, and the third located near the $t t d A$ and $t t d B$ genes for tartrate fermentation [20]. In $K$. pneumoniae 342 (GenBank: CP000964), the oad gene downstream of galETKM is missing while the other two copies were kept. In NTUH-K2044, the oad(dco) genes associated with the 13-kb region as well as the other copy proximal to the galactose metabolism genes were missing; only the copy near the tartrate dehydratase genes was found in the genome. As demonstrated in S. enterica, oxaloacetate decarboxylase is involved in the fermentation of tartrate, presumably following the reaction of tartrate dehydratase, in which tartrate is converted to oxaloacetate $[2,20]$. It is conceivable that the oad genes were recruited to the vicinity of these genes and evolved

Table 2: Detection of the 13-kb genomic region in $187 \mathrm{~K}$. pneumoniae isolates.

\begin{tabular}{|c|c|c|c|c|c|c|}
\hline Specimen type (no. of isolates) & $\begin{array}{l}\text { Primer I } \\
\text { citA, citB }\end{array}$ & $\begin{array}{c}\text { Primer } 2 \\
\operatorname{oad} A, \operatorname{oad} B\end{array}$ & $\begin{array}{l}\text { Primer } 3 \\
\text { citS, citC2 }\end{array}$ & $\begin{array}{c}\text { Primer } 4 \\
\text { citD2, citE2 }\end{array}$ & $\begin{array}{c}\text { Primer } 5 \\
\text { citF2, citG2 }\end{array}$ & Positive* \\
\hline Urine (93) & 56 & 80 & 56 & 58 & 55 & $55(59 \%)$ \\
\hline Non-urine (94) & 54 & 82 & 54 & 54 & 54 & $53(56.3 \%)$ \\
\hline Blood (28) & 18 & 25 & 18 & 18 & 18 & $18(64.2 \%)$ \\
\hline Wound (23) & 11 & 18 & 12 & 12 & 12 & I I (47.8\%) \\
\hline Respiratory (23) & 12 & 20 & 11 & 11 & 11 & II (47.8\%) \\
\hline Other (20) & 13 & 19 & 13 & 13 & 13 & $13(65 \%)$ \\
\hline
\end{tabular}

* Note: Strains with positive results using all 5 primer sets are regarded as positive for the 13-kb region. 
into operons dedicated to different metabolic functions. Incorporation of the oadGAB $(d c o C A B)$ genes in the $13-\mathrm{kb}$ region is likely a result of a secondary insertion event after the acquisition of the cit genes in the ancestral microorganism. This is supported by the data that the $\mathrm{G}+\mathrm{C}$ contents of the oad $(d c o)$ genes are apparently higher than the neighbouring orfs (Figure 1).

\section{Conclusion}

This is the first report distinguishing citrate fermentation biotypes of $K$. pneumoniae. It appears that the genomic variation of citrate fermentation genes among these strains might be more extensive than previously thought since only half of the K. pneumoniae clinical isolates we tested carry the 13-kb genomic island for citrate fermentation. The possession of these genes contributes to their adaptation to different nutrient conditions.

\section{Methods}

\section{Klebsiella pneumoniae strains}

Eight $K$. pneumoniae NK strains (NK3, NK5, NK6, NK8, NK9, NK25, NK29, and NK245) were collected from the Department of Pathology, National Cheng Kung University (NCKU) Hospital, Tainan, Taiwan $[21,22]$. Nine CMK strains (CMKa01 through 08, and CMKa10) were collected from Chung Shan Medical University Hospital, Taichung, Taiwan. The K. pneumoniae strain CG43 was isolated from Chang Gung Hospital, Taoyuan, Taiwan [23]. Strain NTUH-K2044 was isolated from National Taiwan University Hospital, Taipei, Taiwan [12]. The $188 \mathrm{~K}$. pneumoniae strains used to test the association between the citrate fermentation genes and the sites of infection were randomly selected from a nationwide surveillance of antimicrobial resistance collection (Taiwan Surveillance of Antimicrobial Resistance, TSAR) [24]. These clinical strains were not epidemiologically linked. Species identification of the isolates was confirmed by the conventional biochemical reactions [25] in addition to using Vitek Gram Negative Plus Identification card (bioMeìrieux Vitek, Inc. Hazelwood, MO, USA).

\section{Culture of bacteria}

Artificial urine medium (AUM) used in this study was prepared as previously described [15]. Anaerobic cultivations of the bacteria in AUM were performed at $37^{\circ} \mathrm{C}$ using GasPak $^{\mathrm{TM}}$ EZ Gas Generating Pouch Systems (BD, Franklin Lakes, NJ, USA). GasPak ${ }^{\text {TM }}$ Dry Anaerobic Indicator Strips were used to assure anaerobic condition (BD, Franklin Lakes, NJ, USA). Overnight liquid culture of the bacterial strains was harvested and washed by AUM using mini centrifuge, then serial-diluted to an initial optical density at $600 \mathrm{~nm} \quad\left(\mathrm{OD}_{600}\right)$ of approximately 0.0005 $(10,000 \sim 20,000 \times$ dilution) in AUM. Turbidity of the cultured bacteria was monitored spectrophotometrically at $600 \mathrm{~nm}$.

\section{Gene disruption of the 13-kb genomic cluster}

Disruption of the citS together with the nearby regulatory region between the two divergently positioned operons in NK8 genome was done by a method facilitated by $\lambda$ Red recombinase carried on pKD20 [26]. Two PCR primers (cits-HF: 5'-TTAAATCATC ATGCCGAACA CGATGCTGGC GATGACCAGA TTCCGGGGAT CCGTCGACC-3', citc-HR: 5'-TTTTTTAGCG CTTCGTCATT TCAAAACGAA CTGTATTTCT GTAGGCTGGA GCTGCTTC-3') were used to amplify an aac(3)IV $\left(\right.$ Apra $\left.^{\mathrm{R}}\right)$ apramycin resistance gene from pIJ773 [27] while creating the flanking homologous sequence for recombination. As a result, 39-bp from the left end of the citS to the beginning of the citC2 (corresponding to location 3460436125 of the MGH 78578) were disrupted by the apramycin resistant gene in NK8. The gene disruption was confirmed by PCR and DNA sequencing of the corresponding genomic region.

\section{Detection of citrate fermentation genes}

Comparative genomic hybridization (CGH) array (NimbleGen Systems, WI, USA) with probes designed according to the predicted coding sequences spanning the 13-kb genomic region of the K. pneumoniae strain NK8 (with $99 \%$ sequence identity in average compared to syntenic region of MGH 78578) was used to detect differences of this genomic region among the K. pneumoniae clinical isolates. A total of 687 probes were designed isothermally (Tm-balanced) with NimbleGen algorithms across these concatenated CDSs sequences in length of 50-mer with 33-nucleotide overlap between adjacent probe sequences. An intact ribosomal RNA gene cluster (containing 16S23S-5S rRNAs) was included as a positive control. DNA labelling and hybridization methods of genomic DNA, and signal scanning procedure were performed according to manufacturer's instructions. PCR detections of citrate fermentation genes among other clinical isolates were performed using specific primers listed in Table 1 following standard protocols.

\section{DNA sequence}

The complete genomic sequence of $K$. pneumoniae strain NTUH-K2044 has been deposited to the GenBank (accession no. AP006725)[12]. A fosmid clone, KPA-F06C06, containing the $13-\mathrm{kb}$ citrate fermentation gene region, was selected from a fosmid library of $K$. pneumoniae strain NK8.

\section{Authors' contributions}

YTC, TLL ${ }^{3}$, and SFT drafted the manuscript. YTC, and TLL designed and carried out the functional analyses. KMW, TLL 1 , YML, and HYS performed closure/finishing of the genome sequence. TLL ${ }^{3}$, IWH, JJY, MCL, YCL and JTW collected and classified the bacterial strains. YTC, TLL ${ }^{1}$, $\mathrm{KMW}, \mathrm{TLL}^{3}$, and SFT analyzed the data. TLL ${ }^{3}, \mathrm{JJY}, \mathrm{MCL}$, YCL, IJH, JTW, and SFT contributed reagents/materials/ 
analysis tools and participated in design and coordination of the study. YTC, KMW, HYS, and SFT performed annotation. All authors have read and approved the final manuscript.

\section{Acknowledgements}

The project was funded by a grant from the National Science Council (NSC 96-3 I I2-B-400-006) and an intramural grant from the National Health Research Institutes (MG-096-PP09).

\section{References}

I. Schwarz E, Oesterhelt D: Cloning and expression of Klebsiella pneumoniae genes coding for citrate transport and fermentation. EMBO J 1985, 4:1599-1603.

2. Bott $M$ : Anaerobic citrate metabolism and its regulation in enterobacteria. Arch Microbiol 1997, 167:78-88.

3. Kaspar S, Perozzo R, Reinelt S, Meyer M, Pfister K, Scapozza L, Bott $M$ : The periplasmic domain of the histidine autokinase CitA functions as a highly specific citrate receptor. Mol Micorbiol 1999, 33:858-972.

4. Meyer $M$, Dimroth $P$, Bott M: Catabolite repression of the citrate fermentation genes in Klebsiella pneumoniae: Evidence for involvement of cyclic AMP receptor protein. J Bacteriol 200I, I 83:5248-5256.

5. Bott M, Meyer M, Dimroth P: Regulation of anaerobic citrate metabolism in Klebsiella pneumoniae. Mol Microbiol 1995, I 8:533-546

6. Meyer $M$, Dimroth $P$, Bott $M$ : In vitro binding of the response regulator CitB and of its carboxy-terminal domain to $A+T$ rich DNA target sequences in the control region of the divergent citC and citS operons of Klebsiella pneumoniae. J Mol Biol |997, 269:7|9-73|.

7. Schneider K, Kästner CN, Meyer M, Wessel M, Dimroth P, Bott M: Identification of a gene cluster in Klebsiella pneumoniae which includes citX, a gene required for biosynthesis of the citrate lyase prosthetic group. J Bacteriol 2002, 1 84:2439-2446.

8. Johnson JR: Virulence factors in Escherichia coli urinary tract infection. Clin Microbiol Rev I991, 4:80-I28.

9. Bergsten G, Wullt B, Svanborg C: Escherichia coli, fimbriae, bacterial persistence and host response induction in the human urinary tract. Int J Med Microbiol 2005, 295:487-502.

10. Purcell BK, Clegg S: Construction and expression of recombinant plasmids encoding type I fimbriae of a urinary Klebsiella pneumoniae isolate. Infect Immun I983, 39: I I 22- I I 27.

II. Jones CH, Pinkner JS, Roth R, Heuser J, Nicholes AV, Abraham SN, Hultgren SJ: Fim $\mathbf{H}$ adhesin of type I pili is assembled into a fibrillar tip structure in the Enterobacteriaceae. Proc Natl Acad Sci USA 1995, 92:208I-2085.

12. Wu KM, Li LH, Yan JJ, Tsao N, Liao TL, Tsai HC, Fung CP, Chen HJ, Liu YM, Wang JT, Fang CT, Chang SC, Shu HY, Liu TT, Chen YT, Shiau YR, Lauderdale TL, Su IJ, Kirby R, Tsai SF: Genome sequencing and comparative analysis of Klebsiella pneumoniae NTUH-K a strain causing liver abscess and meningitis. J Bacteriol 2044, 1 91:4492-4501.

13. Chou HC, Lee CZ, Ma LC, Fang CT, Chang SC, Wang JT: Isolation of a chromosomal region of Klebsiella pneumoniae associated with allantoin metabolism and liver infection. Infect Immun 2004, 72:3783-3792.

14. Fouts DE, Tyler HL, DeBoy RT, Daugherty S, Ren Q, Badger JH, Durkin AS, Huot H, Shrivastava S, Kothari S, Dodson RJ, Mohamound Y, Khouri H, Roesch LF, Krogfelt KA, Struve C, Triplett EW, Methé BA: Complete genome sequence of the N2-fixing broad host range endophyte Klebsiella pneumoniae 342 and virulence predictions verified in mice. PLoS Genet 2008, 4: el000I4I.

15. Brooks T, Keevil CW: A simple artificial urine for the growth of urinary pathogens. Lett Appl Microbiol I997, 24:203-206.

16. Touchon M, Hoede C, Tenaillon O, Barbe V, Baeriswyl S, Bidet P, Bingen $E$, Bonacorsi S, Bouchier C, Bouvet O, Calteau A, Chiapello $H$, Clermont O, Cruveiller S, Danchin A, Diard M, Dossat C, Karoui ME, Frapy E, Garry L, Ghigo JM, Gilles AM, Johnson J, Le Bouguénec C, Lescat M, Mangenot S, Martinez-Jéhanne V, Matic I, Nassif X, Oztas S, Petit MA, Pichon C, Rouy Z, Ruf CS, Schneider D, Tourret J, Vacherie B, Vallenet D, Médigue C, Rocha EP, Denamur E: Organised genome dynamics in the Escherichia coli species results in highly diverse adaptive paths. PLoS Genet 2009, 5:e I 000344.

17. Brisse S, Fevre C, Passet V, Issenhuth-Jeanjean S, Tournebize R, Diancourt L, Grimont P: Virulent clones of Klebsiella pneumoniae: identification and evolutionary scenario based on genomic and phenotypic characterization. PLOS One 2009, 4:e4982.

18. Giannakopoulos X, Evangelou A, Kalfakakou V, Grammeniatis E, Papandropoulos I, Charalambopoulos K: Human bladder urine oxygen content: implications for urinary tract diseases. Int Urio Nephrol 1997, 29:393-40I.

19. Pos KM, Dimroth P, Bott M: The Escherichia coli citrate carrier CitT: a member of a novel eubacterial transporter family related to the 2-oxoglutarate/malate translocator from spinach chloroplasts. J Bacteriol 1998, I80:4160-4165

20. Woehlke G, Dimroth P: Anaerobic growth of Salmonella typhimurium on ${ }_{\mathrm{L}}(+)-$ and ${ }_{\mathrm{D}}(-)$-tartrate involves an oxaloacetate decarboxylase Na+ pump. Arch Microbiol 1994, I 62:233-237.

21. Chen YT, Shu HY, Li LH, Liao TL, Wu KM, Shiau YR, Yan J], Su IJ, Tsai SF, Lauderdale TL: Complete nucleotide sequence of pK245, a 98-kilobase plasmid conferring quinolone resistance and extended-spectrum-beta-lactamase activity in a clinical Klebsiella pneumoniae isolate. Antimicrob Agents Chemother 2006, 50:386I-3866.

22. Chen YT, Lauderdale TL, Liao TL, Shiau YR, Shu HY, Wu KM, Yan J, Su IJ, Tsai SF: Sequencing and comparative genomic analysis of pK29, a 269-kilobase conjugative plasmid encoding CMY-8 and CTX-M-3 beta-lactamases in Klebsiella pneumoniae. Antimicrob Agents Chemother 2007, 5 I:3004-3007.

23. Chen YT, Chang HY, Lai YC, Pan CC, Tsai SF, Peng HL: Sequencing and analysis of the large virulence plasmid pLVPK of Klebsiella pneumoniae CG43. Gene 2004, 337:189-198.

24. Lauderdale TL, Clifford McDonald L, Shiau YR, Chen PC, Wang HY, Lai JF, Ho M, TSAR Participating Hospitals: The status of antimicrobial resistance in Taiwan among gram-negative pathogens: the Taiwan surveillance of antimicrobial resistance (TSAR) program, 2000. Diagn Microbiol Infect Dis 2004, 48:211-219.

25. Farmer JJ III: Enterobacteriaceae: introduction and identification, In Manual of clinical microbiology. Edited by: Murray PR, Baron EJ, Pfaller MA, Tenover FC, Yolken RH. American Society for Microbiology, Washington, D.C; 1995:438-449.

26. Datsenko KA, Wanner BL: One-step inactivation of chromosomal genes in Escherichia coli K-I 2 using PCR products. Proc Natl Acad Sci USA 2000, 97:6640-6645.

27. Gust B, Challis GL, Fowler K, Kieser T, Chater KF: PCR-targeted Streptomyces gene replacement identifies a protein domain needed for biosynthesis of the sesquiterpene soil odor geosmin. Proc Natl Acad Sci USA 2003, I00: I54 I-I546.

Publish with Bio Med Central and every scientist can read your work free of charge

"BioMed Central will be the most significant development for disseminating the results of biomedical research in our lifetime. "

Sir Paul Nurse, Cancer Research UK

Your research papers will be:

- available free of charge to the entire biomedical community

- peer reviewed and published immediately upon acceptance

- cited in PubMed and archived on PubMed Central

- yours - you keep the copyright
BioMedcentral 